\title{
Fabrication, optimization, and characterization of umbelliferone $\beta$-D-galactopyranoside-loaded PLGA nanoparticles in treatment of hepatocellular carcinoma: in vitro and in vivo studies
}

\author{
This article was published in the following Dove Press journal: \\ International Journal of Nanomedicine \\ II September 2017 \\ Number of times this article has been viewed
}

\begin{abstract}
Vikas Kumar,' Prakash Chandra Bhatt, ${ }^{2}$ Mahfoozur Rahman, Gaurav Kaithwas, ${ }^{3}$ Hani Choudhry, ${ }^{4,5}$ Fahad A Al-Abbasi, ${ }^{4}$ Firoz Anwar, ${ }^{4}$ Amita Verma ${ }^{6}$

'Natural Product Drug Discovery Laboratory, Department of Pharmaceutical Sciences, Faculty of Health Sciences, Sam Higginbottom University of Agriculture, Technology \& Sciences, Allahabad, Uttar Pradesh, India; ${ }^{2}$ Centre for Advanced Research in Pharmaceutical Sciences, Microbial and Pharmaceutical Biotechnology Laboratory, Faculty of Pharmacy, Jamia Hamdard, New Delhi, India; ${ }^{3}$ Department of Pharmaceutical Sciences, Babasaheb Bhimrao Ambedkar University (Central University), Vidya Vihar, Rai Bareli Road, Lucknow, India; ${ }^{4}$ Department of Biochemistry, Faculty of Science, King Abdulaziz University, Jeddah, Saudi Arabia; ${ }^{5}$ Cancer Metabolism and Epigenetic Unit, King Fahd Center for Medical Research, King Abdulaziz University, Jeddah, Saudi Arabia; ${ }^{6}$ Bio-organic and Medicinal Chemistry Research Laboratory, Department of Pharmaceutical Sciences, Faculty of Health Sciences, Sam Higginbottom University of Agriculture, Technology \& Sciences, Allahabad, Uttar Pradesh, India
\end{abstract}

Correspondence: Vikas Kumar Natural Product Drug Discovery Laboratory, Department of Pharmaceutical Sciences, Faculty of Health Sciences, Sam Higginbottom University of Agriculture, Technology \& Sciences, Rewa Road, Naini, Allahabad, Uttar Pradesh 211007, India

Email phvikas@gmail.com

Firoz Anwar

Department of Biochemistry, Faculty of Science, King Abdulaziz University, PO Box 80203, Jeddah 21589, Saudi Arabia Email firoz_anwar2000@yahoo.com
Abstract: Umbelliferone $\beta$-D-galactopyranoside (UFG), isolated from plants, exhibits promising inhibitory action on numerous diseases. The present research was initiated to develop a suitable delivery system for UFG with an intention to enhance its therapeutic efficacy against diethyl nitrosamine (DEN)-induced hepatocellular carcinoma (HCC) in Wistar rats. UFG-loaded polymeric nanoparticles prepared by sonication were scrutinized for average size, drug loading capacity, zeta potential, and drug release potency in animals. HCC cell lines HuH-7 and Hep G2 were used for in vitro cytotoxic investigation. Several hepatic, nonhepatic, antioxidant, and anti-inflammatory biochemical parameters were estimated to establish the anticancer potential of UFG nanoformulation. Microscopical and histopathological investigations were also undertaken to substantiate the results of our work. Umbelliferone $\beta$-D-galactopyranoside-loaded poly(d,1-lactide-co-glycolide) nanoparticles (UFG-PLGA-NP) with particle size of $187.1 \mathrm{~nm}$ and polydispersity index 0.16 were uniform in nature with $82.5 \%$ release of the total amount of drug after $48 \mathrm{~h}$. Our study successfully established the development and characterization of UFG-PLGA-NP with noticeable effect against both in vivo and in vitro models. The anticancer potential of UFG-PLGA-NP was brought about by the management of DEN-induced reactive oxygen species generation, mitochondrial dysfunction, proinflammatory cytokines alteration, and induction of apoptosis. Positive zeta potential on the surface of UFG-PLGA-NP would have possibly offered higher hepatic accumulation of UFG, particularly in the electron-dense mitochondria organelles, and this was the take-home message from this study. Our results demonstrated that such polymer-loaded delivery systems of UFG can be a better option and can be further explored to improve the clinical outcomes against hepatic cancer.

Keywords: hepatocellular carcinoma, umbelliferone $\beta$-D-galactopyranoside nanoparticles, diethyl nitrosamine, cytokines, oxidative stress

\section{Introduction}

Hepatocellular carcinoma (HCC) is one of the leading risks to human health, the fifth most common cancer worldwide, and is ranked third in causing cancer-related deaths. Hepatitis $\mathrm{C}$ virus, ${ }^{1}$ hepatitis $\mathrm{B}$ virus, ${ }^{2}$ Aflatoxin $\mathrm{B}_{1}$, and diethyl nitrosamine (DEN) are the prime risk factors for $\mathrm{HCC}^{3}$ Past efforts have brought very small success in treatment of HCC patients. The available option for treatment of HCC is liver transplantation and surgical resection, which can only be beneficial for patients where early detection or diagnosis is made to detect this disease. Hormonal therapy, radiotherapy, and immune-chemotherapy are the other options for $\mathrm{HCC}$ treatment but 
with limitations like tumor shapes, type, number, stage, and vascular movement along with patient's medical history for successful outcome. These therapies are always accompanied by higher toxicity and drug resistance and generally affect the normal cells along with cancer cells. The related side or adverse effects such as diarrhea, thrombocytopenia, constipation, alopecia, and nausea overshadow the treatment and debilitate the patient. The limitations of the drugs currently available for HCC keyed the researchers to take new approach for higher clinical efficacy with minimized side effects. ${ }^{4}$ Therefore, researchers have become more focused on natural drugs for cancer prevention including the treatment of HCC. In this effort, some natural compounds showed promising results with striking success in preclinical setup, but poor bioavailability and systemic toxicity were the major hurdles. ${ }^{5}$ Various experimental and clinical studies have proved the importance of free radical scavenger-rich food that can reduce or minimize the chronic diseases, especially cancer. 7-hydroxycoumarin (umbelliferone) already proved for its antidiabetic, anti-inflammatory, and antinociceptive effect via free radical scavenging along with positive potential on arthritis, laryngeal cancer, renal cancer, and airway inflammation. These major breakthroughs revoked us to make an effort in evaluating umbelliferone for its anticancer effect against DEN-induced HCC.

Although it is well documented that umbelliferone has significant effect in restricting the proliferation and cytotoxic potential in liver cells, no study was undertaken for its nanoformulation. Nanoformulation provides an alternative form of drug delivery system, which attributes to the feasibility of incorporating hydrophilic and hydrophobic molecules, better cellular uptake, and long shelf-life of molecules. ${ }^{6,7}$ Poly(d,l-lactide-co-glycolide) (PLGA) is a biodegradable polymer, regularly used as a biomaterial for its outstanding biocompatibility, along with its use in drug delivery systems, including films, blends, microspheres, matrixes, pellets, and nanoparticles, to name few. ${ }^{8}$ Various studies have showed that polymeric nanoparticles of naturally occurring compounds, namely, curcumin and quercetin, have enhanced significant chemopreventive potential against various disorders. ${ }^{7,8}$ Hence, it was thought to be worth scrutinizing the effectiveness of umbelliferone $\beta$-D-galactopyranoside (UFG) and its polymeric nanoparticles formulation in combating DEN-induced hepatocarcinogenesis.

\section{Materials and methods}

\section{Chemicals and equipments}

UFG was isolated by Kumar et al ${ }^{9,10}$ All the chemicals and reagents used for the preparation of formulation were procured from Hi media, India. The polydispersity index (PDI) and particle size (PS) of the nanoformulation were determined using the Zeta Size Nao ZS (Malvern Instruments Limited, Worcestershire, UK). The LEO $435 \mathrm{~V}$ scanning electron microscopy (SEM) (Leo Electron Microscopy Ltd., Cambridge, UK) and transmission electron microscopy (TEM) CM-10 (Philips, the Netherlands) were used for the microscopical analysis of formulation. Radioactivity was measured by a gamma ray spectrometer (GRS23C, Electronics Corporation of India Limited, India).

\section{Formulation of UFG polymeric nanoparticles}

Double emulsion solvent techniques with minor modification were used for the preparation of polymeric nanoparticles by Perlatti et al. ${ }^{11}$ Dispersing agents Tween 80 and Span 60 were employed for the development of internal and external aqueous phase with polyvinyl alcohol (PVA) as external aqueous phase and stearic acid as the stabilizing agent. Primary emulsion was sonicated by an ultrasonicator under ice-cool bath for $15 \mathrm{~s}$, just to prevent the elevation of temperature, and the resultant mixture was gradually deionized by PVA solution $(5 \mathrm{~mL})$ with or without surfactant (Tween 80 ) in various concentrations. The above mixture was again sonicated for $15 \mathrm{~s}$ for secondary emulsion $\left(\mathrm{W}_{1} / \mathrm{O} / \mathrm{W}_{2}\right)$ with continuous stirring at 1,200 rpm for complete evaporation of dichloromethane. Residual surfactants and free drug were removed from polymeric nanoparticles by successive washing. Nanoparticles were stabilized by dispersing them in a low volume of water and lyophilized for $24 \mathrm{~h}$. A similar protocol was followed for blank nanoparticles and control group, and were kept at $-20^{\circ} \mathrm{C}$ for further use (Figure 1).

\section{Optimization of formulation parameters using Box-Behnken design (BBD)}

Drug loading (DL) concentration, PLGA polymer concentration, and sonication time were analyzed for umbelliferone $\beta$-D-galactopyranoside-loaded PLGA nanoparticles (UFG-PLGA-NP). Formations of polymeric nanoparticles were evaluated for the above factors with the use of response surface methodology using BBD and Design Expert Software 8.0.7.1. All the experiments were performed in triplicate. Seventeen runs were performed with three independent factors (DL, polymer concentration, and sonication time) from BBD to obtain a three-level factorial design of experiments (Table 1). Response surface and contour plots were generated for better understanding of interaction between the response variables and the independent factors. Numerical and graphical optimizations were performed to 


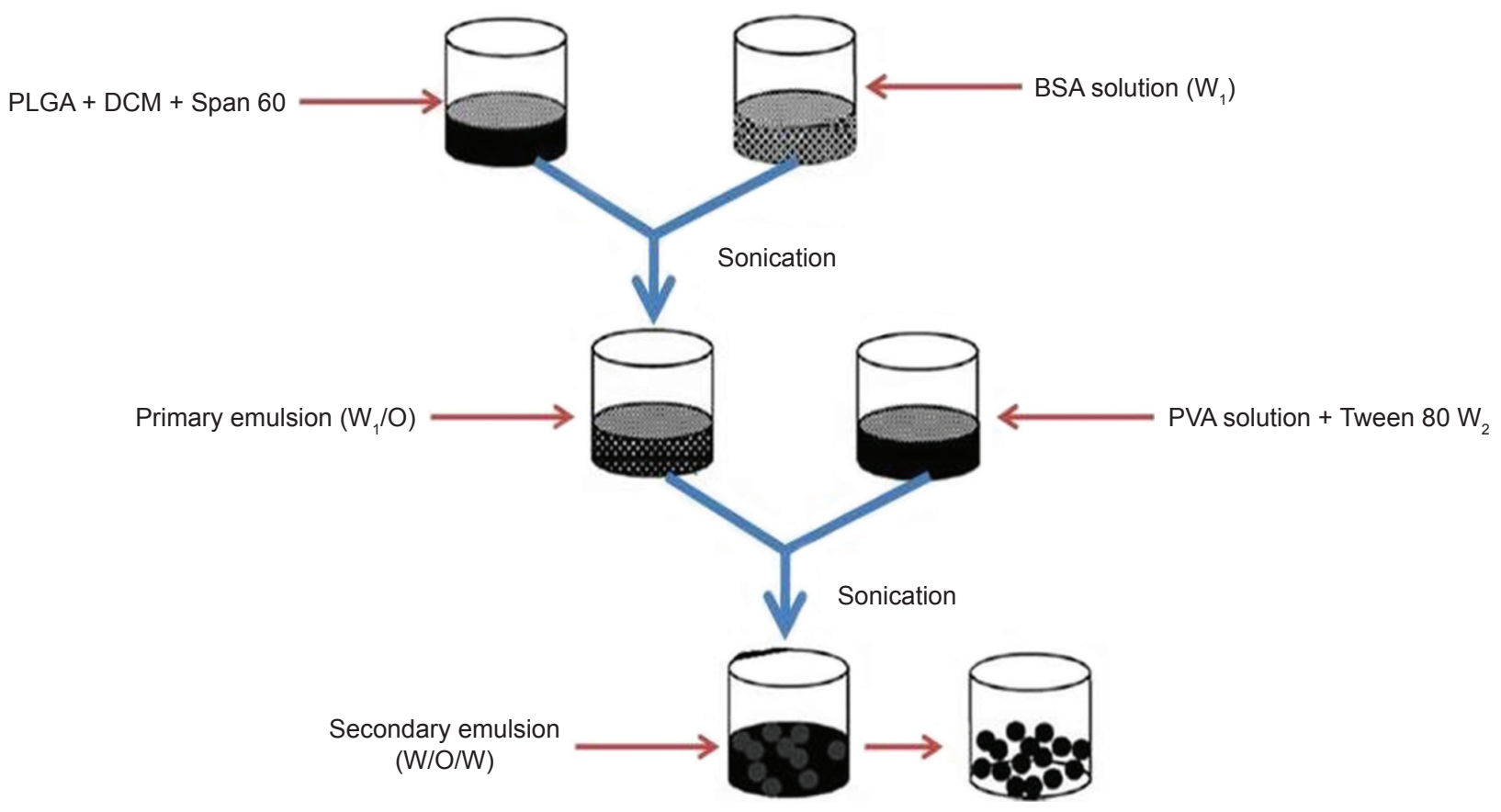

Figure I Schematic representation of development of umbelliferone $\beta$-D-galactopyranoside polymeric nanoformulation. Abbreviations: BSA, bovine serum albumin; DCM, dichloromethane; PLGA, poly(d,I-lactide-co-glycolide); PVA, polyvinyl alcohol.

determine the optimum conditions for preparation of UFGPLGA-NP and the predicted values of desired responses.

\section{Characterization of UFG-PLGA-NP} Estimation of PS and PDI of UFG-PLGA-NP

PS and PDI of UFG-PLGA-NP were analyzed by Zetasizer (Nano-ZS, Malvern Instruments). The prepared UFGPLGA-NP were harvested and washed with deionized water to remove free drug, free polymer, and surfactants. The particles were dispersed in a low volume of water and lyophilized for $24 \mathrm{~h}$ to obtain powder and stable nanoparticles. The prepared formulation was kept at $-20^{\circ} \mathrm{C}$. Estimation of PS and PDI was made by diluting the nanoformulation with deionized water (200 times) followed by constant shaking to obtain about $100-250$ kilo counts per second. Dynamic light scattering technique was used for the determination of PDI and PS of the UFG-PLGA-NP.

\section{Release of UFG-PLGA-NP}

Dialysis technique was employed to determine the release of UFG-PLGA-NP by phosphate buffer $(\mathrm{pH}=7.4)$. The aliquots obtained at each point of time were analyzed for drug released as a function of time.

\section{Entrapment efficiency and DL of UFG- PLGA-NP}

The loading capacity and entrapment efficiency (EE) of UFG-PLGA-NP were estimated by separating free UFG from the PLGA-NP at 11,000 rpm for 30 min centrifugation. The free UFG present in the supernatant was quantified

Table I Effect of UFG-PLGA-NP treatment on the liver parameters in DEN-induced carcinogenesis in rats

\begin{tabular}{|c|c|c|c|c|c|c|c|}
\hline S no & Groups & $\begin{array}{l}\text { Initial body } \\
\text { weight (g) }\end{array}$ & $\begin{array}{l}\text { Final body } \\
\text { weight (g) }\end{array}$ & $\begin{array}{l}\text { Liver } \\
\text { weight }(g)\end{array}$ & $\begin{array}{l}\text { Relative liver weight } \\
\text { (g liver/1 } 00 \text { g body weight) }\end{array}$ & iPH & PG \\
\hline $\mathrm{I}$ & Normal control & $180.2 \pm 2.38$ & $435.6 \pm 5.12$ & $10 \pm 1.05$ & $2.29 \pm 0.34$ & 0.967 & 58.63 \\
\hline 2 & Normal control + UFG $(50 \mathrm{mg} / \mathrm{kg})$ & $176.8 \pm 2.48$ & $441.2 \pm 7.99$ & $9.6 \pm 1.12$ & $2.17 \pm 0.45$ & 0.957 & 59.93 \\
\hline 3 & DEN control & $183.4 \pm 2.73$ & $342.8 \pm 22.96 \ldots$ & $15.2 \pm 2.83^{\# \# \# ~}$ & $4.43 \pm 0.99 \ldots \ldots$ & 0.976 & 46.49 \\
\hline 4 & DEN control + UFG (5 mg/kg) & $\mid 78.4 \pm 1.42$ & $377.2 \pm 16.56^{*}$ & $14.4 \pm 1.98^{*}$ & $3.82 \pm 0.75^{*}$ & 0.962 & 52.70 \\
\hline 5 & DEN control + UFG $(50 \mathrm{mg} / \mathrm{kg})$ & $183.6 \pm 2.63$ & $403 \pm 13.04 * *$ & $13.8 \pm 1.23 * *$ & $3.42 \pm 0.23 * *$ & 0.976 & 54.44 \\
\hline 6 & DEN control + UFG-PLGA-NP & $192.6 \pm 3.75$ & $428.2 \pm 12.32 * * *$ & $11.8 \pm 1.33^{* * *}$ & $2.76 \pm 0.34^{* * *}$ & I & 55.02 \\
\hline
\end{tabular}

Notes: DEN control group compared with normal control group. $P<0.001$ is considered as extremely significant and the treated groups compared with the $D E N$ control. $* P<0.05$ is considered as significant, $* * P<0.01$ is considered as very significant, $* * * P<0.001$ is considered as extremely significant. Statistical analysis was performed by oneway analysis of variance followed by the Dunnett's test. Data presented as mean $\pm S D$.

Abbreviations: DEN, diethyl nitrosamine; iPH, ponderal homogeneity index; PG, ponderal grain; PLGA, poly(d,I-lactide-co-glycolide); S no, serial number; UFG, umbelliferone $\beta$-D-galactopyranoside; UFG-PLGA-NP, UFG-loaded PLGA nanoparticles. 
through high-performance liquid chromatography. The EE and DL capacity of the UFG-PLGA-NP were calculated by calculating the difference between the initial amount of UFG used in formulation and the amount of UFG present in supernatant after centrifugation at $21 \mathrm{~g} \mathrm{rpm}$ for $30 \mathrm{~min}$ at $10^{\circ} \mathrm{C}$. EE of the PLGA-NP was determined by the following formula:

$$
\text { EE }(\%)=\frac{\begin{array}{c}
\text { Total amount } \\
\text { of UFG }
\end{array}-\begin{array}{c}
\text { Amount of UFG in } \\
\text { total supernatant }
\end{array}}{\text { Amount of UFG }} \times 100
$$

\section{SEM and TEM of UFG-PLGA-NP}

During the SEM analysis, a small quantity of UFG-PLGA-NP was mounted on an aluminum sample, which was sputtercoated with gold-palladium alloy to reduce the surface charge. LEO 435 V (SEM) (Leo Electron Microscopy Ltd., Cambridge, UK) was used at a $155 \mathrm{~mm}$ working distance and $15 \mathrm{kV}$ accelerating voltage.

Microscopical evaluation was carried by TEM CM-10 (Philips, the Netherlands). In brief, one drop of UFGPLGA-NP formulation was placed on a carbon-coated grid with $2 \%$ phosphotungstic acid and left for $30 \mathrm{~s}$. The coated dried grid was mounted on the slide and observed.

\section{In vitro studies}

\section{Assessment of growth inhibition in HCC cell lines}

Cell lines storage and culture conditions

HCC cell lines HuH-7 and Hep G2 were established by the method reported by Farmer et al. ${ }^{4}$ The cells were cultured by Dulbecco's Modified Eagle's Medium and maintained in an incubator with $\mathrm{CO}_{2}$ with constant humidity. The sensitivity of UFG was determined in both the cell lines.

\section{Preparation of various dilutions of UFG}

The solution of UFG was prepared with serial dilution, mixed with media to obtain the various concentrations of UFG in solution with application of it on growing adherent cells. The dose of the UFG ranging from 0.1 to $50 \mathrm{mM}$ was selected for the in vitro anticancer activity.

\section{Estimation of growth inhibition}

Viable cell counting

The effect of UFG was evaluated on cells by direct counting. Both cell lines (HuH-7 and Hep G2) were seeded into plates (60 $\mathrm{mm}$ ) with different concentrations of UFG loaded on culture media. After $48 \mathrm{~h}$, trypan blue dye exclusion was used for counting the floating and adherent cells. The enhancement of relative rate of cell counts in the presence of ligands was compared with control (without drug) to give the considerable representation of cell growth.

\section{Estimation of DNA synthesis}

${ }^{3} \mathrm{H}$ thymidine incorporation was used for determination of DNA synthesis. Briefly, the cells were harvested into 24-well plates in complete culture media, with various concentrations of UFG in media. ${ }^{4}$ After 24 h, $1 \mu \mathrm{Ci}$ (methyl-3H)-thymidine was mixed in each well and incubated for $6 \mathrm{~h}$. The cells were harvested by glass fiber filter mats and dried for $1 \mathrm{~h}$. The level of ${ }^{3} \mathrm{H}$ thymidine incorporation was estimated by 1450 Microbeta $^{\mathrm{TM}}$ scintillation counter (Wallac Oy, Finland).

\section{Animals}

Swiss Albino Wistar (100-130 g, male) rats were used for the current experimental study. The rats were caged in the animal house under the standard temperature $\left(23^{\circ} \mathrm{C} \pm 5^{\circ} \mathrm{C} ; 60 \% \pm 5 \%\right.$ relative humidity) with $12: 12 \mathrm{~h}$ light and dark cycle. The experimental animals were on standard pellet diet and water ab libitum. The entire protocol was approved by the Institutional Animal Ethical Committee (IAEC) and all of the study was performed in accordance with the guidance of Committee for the Purpose of Control and Supervision of Experiments on Animals, Ministry of Social Justice and Empowerment, Government of India. The current study was approved by the Laboratory Animal Facility, Sam Higginbottom University of Agriculture, Technology \& Sciences, Allahabad (IAEC/ SHIATS/PA151X/FVK01).

\section{Experimental design}

Rats were randomly selected in six groups, which received carcinogen with tested drug as per their individual body weights depicted below.

- Group I: Normal control (untreated controls and received the three doses of olive oil with regular interval between doses)

- Group II: Normal control + UFG (50 mg/kg)

- Group III: DEN control

- Group IV: DEN + UFG (5 mg/kg)

- Group V: DEN + UFG (50 mg/kg)

- Group VI: DEN + UFG-PLGA-NP (containing the $5 \mathrm{mg} / \mathrm{kg}$ UFG).

Group III-VI rats were treated intraperitoneally with three doses of DEN (200 mg/kg, body weight). All the concerned group rats received the oral administration of various doses of UFG and UFG-PLGA-NP, respectively. At the end of the experimental study (22 weeks), the final body weight of rats was estimated, blood collected from the retro-orbital route, liver tissue was excised, cleaned with ice-cold saline, and 
further blotted to dryness. It was homogenized with $0.1 \mathrm{M}$ phosphate buffer saline ( $\mathrm{pH}=7.4)$ and subjected to highspeed centrifugation to obtain clear supernatant component. The clear supernatant was further used for the estimation of biochemical parameters.

\section{Biochemical parameters}

All the hepatic biochemical parameters including aspartate amino transferase (AST), alanine aminotransferase (ALT), and alkaline phosphatase (ALP) were estimated by standard kits (Span Diagnostic Lab). The serum $\alpha$-feto protein (AFP) was determined using the reported method of Anwar et al. ${ }^{6}$ Nonhepatic parameters, namely, total protein, albumin, total bilirubin, and gamma-glutamyl transpeptidase (GGPT), were also estimated by standard reported methods. ${ }^{11-14}$

\section{Hematological parameters}

The hematological parameters such as red blood cells (RBC), erythrocytes sedimentation rate (ESR), white blood cells (WBC), and hemoglobin $(\mathrm{Hb})$ were estimated using the method reported by Kumar et al. ${ }^{15,16}$

\section{Antioxidant parameters}

Antioxidant parameters were estimated from liver homogenates such as catalase (CAT), lipid peroxidation (LPO), superoxide dismutase (SOD), glutathione transferase (GST), glucose 6-phosphate dehydrogenase, conjugated dienes (CD), and glutathione peroxidase (GPx) by standard methods.

\section{Estimation of proinflammatory cytokines}

The proinflammatory cytokines interleukin-1 $\beta$ (IL-1 $\beta$ ), tumor necrosis factor- $\alpha$ (TNF- $\alpha)$, and IL-6 were estimated by kits of enzyme-linked immunosorbent assay as per the manufacturer's instruction.

\section{Isolation and autopsy of tissue}

After 22 weeks of treatment, all rats were sacrificed. After postpartum, liver was excised immediately, cleaned, washed, and rinsed in ice-cold normal saline solution (until bleached of blood) and blotted dry on filter paper to remove blood. A small representative portion of liver ( $\sim 5 \mathrm{~mm}$ thick) was fixed in $10 \%$ formalin and stored at $4^{\circ} \mathrm{C}$.

\section{Statistical analysis}

Design Expert Software was used for the optimization of various independent variables. All the values of in vitro experimental results were represented as mean \pm standard deviation (SD), while in vivo results were shown as mean \pm standard error of mean. Statistical analysis was performed by GraphPad Prism one-way analysis of variance (ANOVA) followed by the Dunnett's test.

\section{Result}

\section{Physicochemical characteristics of UFG- PLGA-NP}

The physiochemical characteristics were defined in terms of PDI, PS, and EE of UFG-PLGA-NP and are summarized in Table S1. TEM and SEM output clarifies that the nanoformulation of UFG-PLGA-NP has regular shape, smooth surface, and PDI, which confirms uniform size distribution. Zeta potential results indicate the stable polymeric NP of UFG-PLGA-NP. In vitro release study illustrated a biphasic pattern with an initial burst during the first $4 \mathrm{~h}$, followed by slow and sustained release of UFG-PLGA-NP.

\section{Characterization and optimization of UFG-PLGA-NP}

Optimization of polymeric nanoformulation of UFG was performed by PDI and PS distribution. The actual and predicted data for PDI and PS are tabulated in Table S2. The data were generated, scrutinized by the design expert software, and predicated for PS, PDI, and EE.

\section{Final equation regarding coded factors for particle size}

$$
\begin{aligned}
\mathrm{PS}= & +184.18+37.86^{*} \mathrm{~A}-65.85^{*} \mathrm{~B}-11.78^{*} \mathrm{C}+2.55^{*} \\
& \mathrm{AB}+5.20^{*} \mathrm{AC}+0.25^{*} \mathrm{BC}+102.48^{*} \mathrm{~A}^{2}+76.07 * \\
& \mathrm{~B}^{2}-26.18^{*} \mathrm{C}^{2}
\end{aligned}
$$

\section{Final equation regarding coded factors for PDI}

$$
\begin{aligned}
\mathrm{PDI}= & +0.20+0.041 * \mathrm{~A}-0.084 * \mathrm{~B}-0.022 * \mathrm{C}-0.013 * \\
& \mathrm{AB}-0.037 * \mathrm{AC}-2.500 \mathrm{E}-004 * \mathrm{BC}+0.11 * \\
& \mathrm{~A}^{2}+0.092 * \mathrm{~B}^{2}-0.044^{*} \mathrm{C}^{2}
\end{aligned}
$$

\section{Final equation regarding coded factors for $E E$}

$$
\begin{aligned}
\mathrm{EE}= & +86.56-3.63 * \mathrm{~A}+13.05^{*} \mathrm{~B}+0.57 * \mathrm{C}+1.57 * \\
& \mathrm{AB}-0.69^{*} \mathrm{AC}+0.59 * \mathrm{BC}-7.22 * \mathrm{~A}^{2}-11.10^{*} \\
& \mathrm{~B}^{2}+0.36^{*} \mathrm{C}^{2}
\end{aligned}
$$

Three-dimensional response surface $(\mathrm{B}, \mathrm{D}$, and $\mathrm{F})$ and contour plots (A, C, and E) illustrate the effects of polymer concentration (A), drug percentage (B), and sonication 
time (C), and the effect of the reciprocal interaction of the dependent variables of the UFG formulation is represented in Figure S1 (PDI), Figure S2 (PS), and Figure S3 (EE), respectively. The ANOVA of the model for PS and PDI is presented in Table S2. Point prediction of design expert software was used to determine the optimum values of formulation factors for PDI and the smaller PS of UFG-PLGA-NP.

\section{In vitro release of UFG-PLGA-NP}

The in vitro release study demonstrated the total amount of drug released after $48 \mathrm{~h}$, which was found to be $82.5 \%$. The release pattern confirmed the controlled and sustained-release study of UFG-PLGA-NP (Figure S4). Figure 2 represents the PS distribution of UFG-PLGA-NP.

\section{UFG induced growth inhibition}

The treatment of UFG on both cell lines after $48 \mathrm{~h}$ showed the significant reduction in the relative increment of cell number at concentrations of 10,25 , and $50 \mu \mathrm{M}$ (Figure $3 \mathrm{~A}$ ).

A

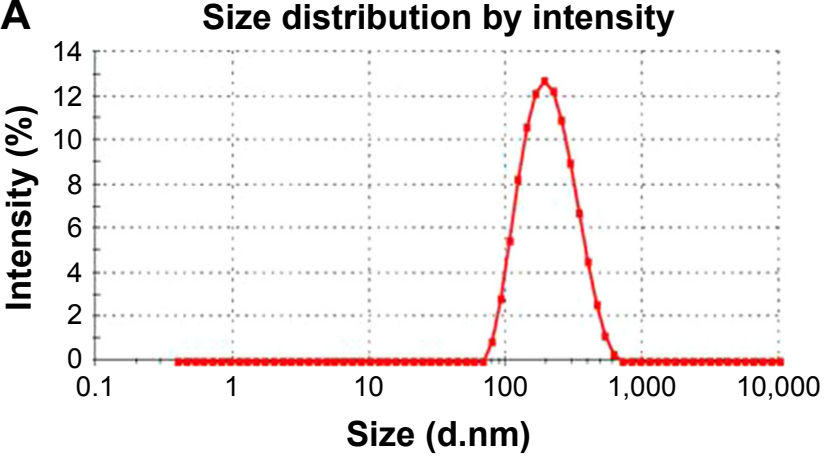

\section{UFG inhibited DNA synthesis}

It was confirmed by estimation of ${ }^{3} \mathrm{H}$ thymidine incorporation in both types of cell lines. Figure 3B confirmed that the UFG significantly inhibited the ${ }^{3} \mathrm{H}$ thymidine incorporation in a dose-dependent manner for both cell lines.

\section{Animal growth, food, and water intake}

Swiss albino Wistar rats were used for the evaluation of hepatocarcinogen effect induced by DEN on liver. However, during the experimental period, none of the rats showed any external sign of disease, except that the environmental cage enrichment to prevent aggressive behavior, sporadic injuries, and focal loss of hair was reported.

Table 1 represents body weight $\mathrm{PH}$ and $\mathrm{PG}$ for the rats of all groups. Normal control group rats showed more water and food consumption as compared to DEN-exposed groups (data not shown). The level of water consumption and food between the normal control and DEN control was not statistically significant.

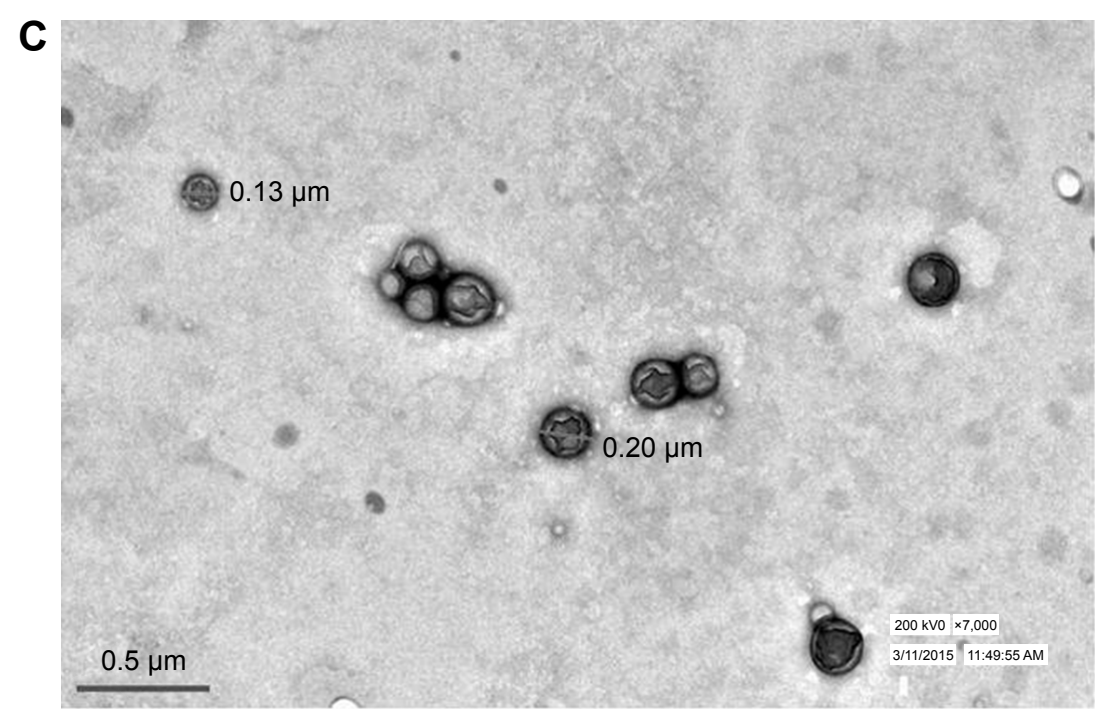

Figure 2 Particle size distribution curve.

Notes: (A) Particle size distribution of prepared PLGA-based polymeric UFG polymeric NP. (B) Scanning electron microscopy of prepared UFG-PLGA-NP. (C) Transmission electron microscopy of prepared PLGA-UFG-NP.

Abbreviations: NP, nanoparticles; PLGA, poly(d,I-lactide-co-glycolide); UFG, umbelliferone $\beta$-D-galactopyranoside; UFG-PLGA-NP, UFG-loaded PLGA NPs.

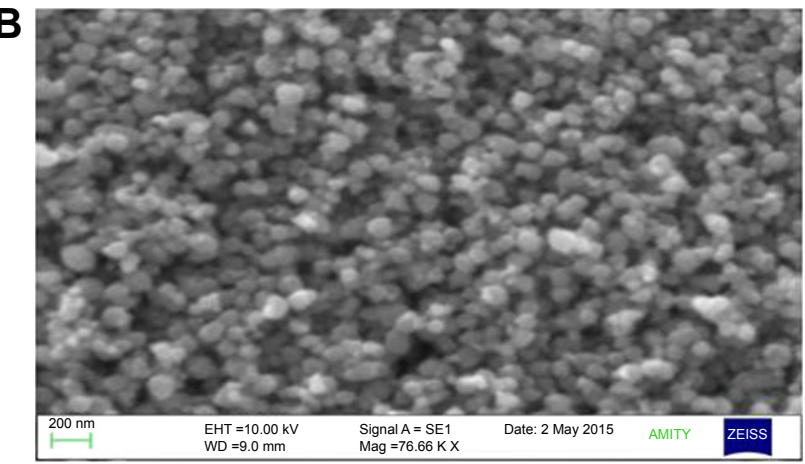


A

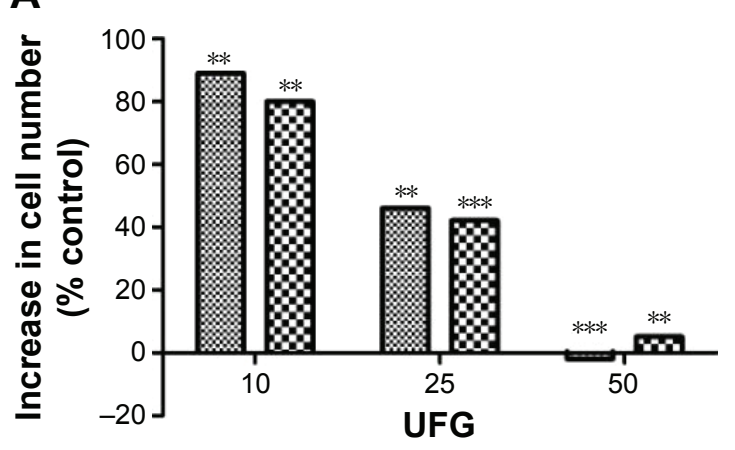

B

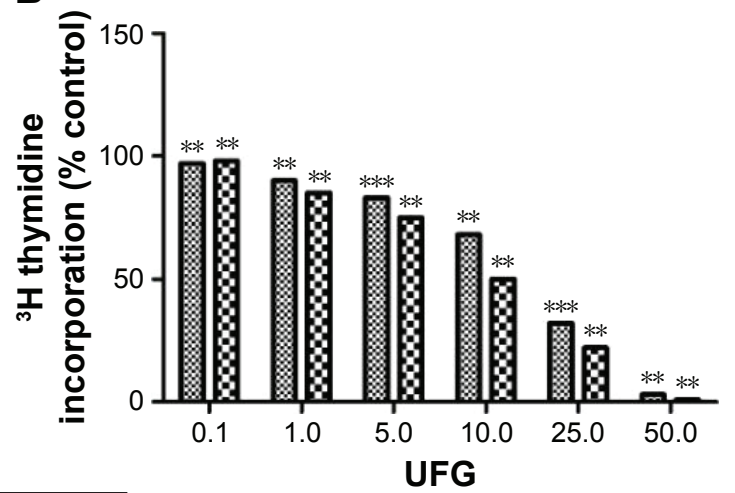

Hep G2 \& HuH-7

Figure 3 Effect of the UFG-PLGA-NP on Hep G2 and HuH-7 cell lines.

Notes: (A) Effect of UFG on proliferation of hepatocellular carcinoma cell lines. Cells were incubated in the presence or absence (control) of UFG, and after $48 \mathrm{~h}$, total number of viable cells was counted by trypan blue dye exclusion. Increment of cell number with drugs is expressed compared with that of control. (B) UFG induced inhibition of DNA synthesis in hepatocellular carcinoma cell lines. Cells were treated with UFG in $0.1-50 \mu \mathrm{M}$ concentration, and after $24 \mathrm{~h}$, a standard ${ }^{3} \mathrm{H}$ thymidine incorporation assay was performed. All the values are given as mean \pm standard error of the mean. $* * P<0.01$ is considered as significant; $* * * P<0.00 \mathrm{I}$ is considered as very significant. Abbreviations: UFG, umbelliferone $\beta$-D-galactopyranoside; UFG-PLGA-NP, UFG-loaded poly(d,I-lactide-co-glycolide) nanoparticles.

\section{Effect of UFG and UFG-PLGA-NP on body weight of DEN-induced $\mathrm{HCC}$ rats}

As shown in Table 1, the body weight of UFG and DEN group animals was significantly $(P<0.001)$ decreased as compared to normal control and normal control + UFG $(50 \mathrm{mg} / \mathrm{kg})$. The normal control group rats and normal control + UFG $(50 \mathrm{mg} / \mathrm{kg})$ group rats showed the lower liver/body weight ratio as compared to DEN control, and UFG-, and UFGPLGA-NP-treated rats. However, the liver weight of DEN group rats was significantly higher than another group's rats.

\section{Effect of UFG and UFG-PLGA-NP on liver nodules in DEN-induced hepatocar- cinogenesis}

Table $\mathrm{S} 3$ epitomized the total number of rats, liver nodules, nodule incidences, and an average number of nodules/ nodule-bearing liver (nodule multiplicity). DEN made upsurge evident in development of hepatic nodules, while there were no visible nodules observed in the normal control and normal control + UFG (50 mg/kg) group. DEN-induced hepatocarcinogenesis rats treated with UFG declined the hepatic nodules in a dose-dependent manner. DEN-induced rats treated with UFG-PLGA-NP decreased the hepatic nodules as compared to DEN control group rats. UFG-PLGA-NP significantly declined the hepatic nodules as compared to DEN-induced control group rats (Table S4).

\section{Effect of UFG and UFG-PLGA-NP on liver parameter}

DEN made evident to boost the level of AST (155.4 \pm 3.012$)$, ALP (80 \pm 2.025$)$, ALT (133.4 \pm 2.638$)$, and AFP (71.4 \pm 1.887$)$.
DEN-induced hepatocarcinogenesis rats treated with UFG-PLGA-NP bestow an imperative fortification in a dose-dependent manner (Figure S5).

\section{Effect of UFG and UFG-PLGA-NP on non-liver parameters}

HCC rats showed the decreased level of total protein, albumin, GGPT, and total bilirubin (Figure S6) along with the descanted level of albumin and total protein, which implied the destitute liver functions and failure to fight infections. These rats treated with UFG-PLGA-NP significantly elevated the level of total protein, albumin, GGPT, and total bilirubin as compared to other groups of treatment. The elevated levels of total protein, albumin, and $\mathrm{A} / \mathrm{G}$ indicate the reform of liver function in DEN-induced hepatocarcinogenesis.

\section{Effect of UFG and UFG-PLGA-NP on hematological profile}

We further estimated the effect of DEN and UFG on the hematological parameters such as WBC, RBC, ESR, and $\mathrm{Hb}$ (Figure S7). DEN control group confirmed the boosted content of WBC and ESR and reduced level of RBC and $\mathrm{Hb}$, which were significantly $(P<0.001)$ restored by the UFG. However, DEN-induced rats treated with UFG-PLGA-NP significantly $(P<0.001)$ modulated the hematological profile near to the normal control group rats.

\section{Effect of UFG and UFG-PLGA-NP on antioxidant enzymes}

Diseased rats confirmed the increased level of LPO, determined in term of malonaldehyde (MDA) as compared to 
normal rats. UFG treatment confirmed the dose-dependent reduction in $\mathrm{LPO}$, supporting the antioxidant effect of drug. UFG-PLGA-NP group rats afforded the momentum of protection against lipid peroxides induced by DEN in hepatic tissue. The extent of CD in the hepatic tissue confirmed the increased level of $C D$, which was significantly $(P<0.001)$ altered by the UFG in a dose-dependent manner. In contrast, rats in the UFG-PLGA-NP-treated group showed a remarkable inhibition in the content of CD as compared to other groups. DEN treatment showed the significant $(P<0.001)$ reduced content of reactive oxygen species (ROS) detoxifying enzymes such as GPx, SOD, glucose-6-phosphate, GST, and CAT, which were significantly $(P<0.001)$ attenuated by UFG in a dose-dependent treatment. The UFG-PLGA-NP treatment confirmed the marked alteration in the content of detoxifying enzymes. The results confirmed that the UFG-PLGA-NP significantly $(P<0.001)$ modulated the DEN-induced alteration in the content of nonenzymatic antioxidant parameters (Figure S8).

\section{Effect of UFG-PLGA-NP on inflammatory mediators}

Figure 4 represents the proinflammatory cytokines such as TNF- $\alpha$, IL-6, and IL-1 $\beta$. The increased level of TNF- $\alpha$ in diseased rats was significantly $(P<0.001)$ reduced by UFGPLGA-NP at the end of the experimental study. Proinflammatory cytokines, namely, IL-6, displayed a similar trend as that of TNF- $\alpha$. DEN group rats showed the enhanced level of IL-6 as compared to normal group rats. Rats in the UFGPLGA-NP-treated group showed the significant $(P<0.001)$ decline in IL-6 level. DEN-induced rats showed the increased level of IL-1 $\beta$ as compared to normal control, which was significantly $(P<0.001)$ restored by UFG-PLGA-NP.

\section{Effect of UFG-PLGA-NP on the histopathology}

Histopathological changes of normal, and DEN- and UFGPLGA-NP-treated rats are well defined in Figure 5. Normal control group rats showed the normal, typical structure distinguished with normal architecture with small uniform nuclei,
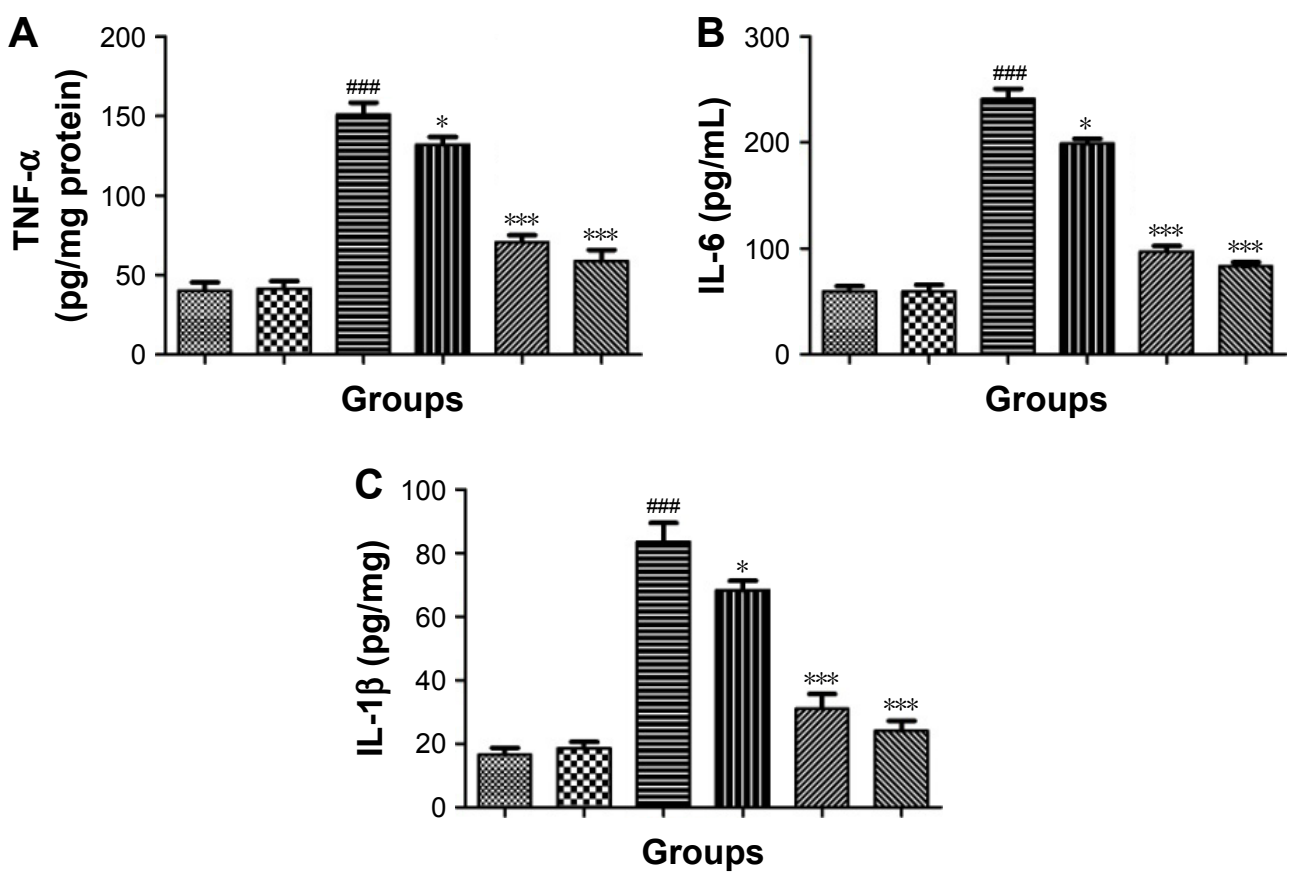

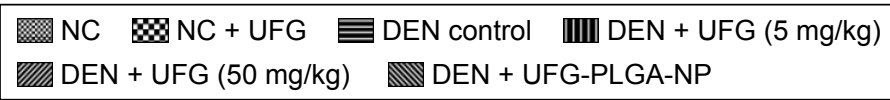

Figure 4 Effect of UFG-PLGA-NP on proinflammatory cytokines in DEN-induced hepatocarcinogenesis rat.

Notes: Proinflammatory cytokines were assessed in terms of altered level of (A) TNF- $\alpha$, (B) IL-6, and (C) IL-I $\beta$ as described in the "Materials and methods" section. DEN control group compared with normal control group. ${ }^{\# P<0.00 I}$ is considered as extremely significant and the treated groups compared with the DEN control. $* P<0.05$ is considered as significant and ${ }^{*} * \mathrm{P}<0.00 \mathrm{I}$ is considered as extremely significant. Statistical analysis was performed by one-way analysis of variance followed by the Dunnett's test.

Abbreviations: DEN, diethyl nitrosamine; IL-I $\beta$, interleukin I beta; IL-6, interleukin-6; NC, normal control; PLGA, poly(d,I-lactide-co-glycolide); TNF- $\alpha$, tumor necrosis factor- $\alpha$; UFG, umbelliferone $\beta$-D-galactopyranoside; UFG-PLGA-NP, UFG-loaded PLGA nanoparticles. 

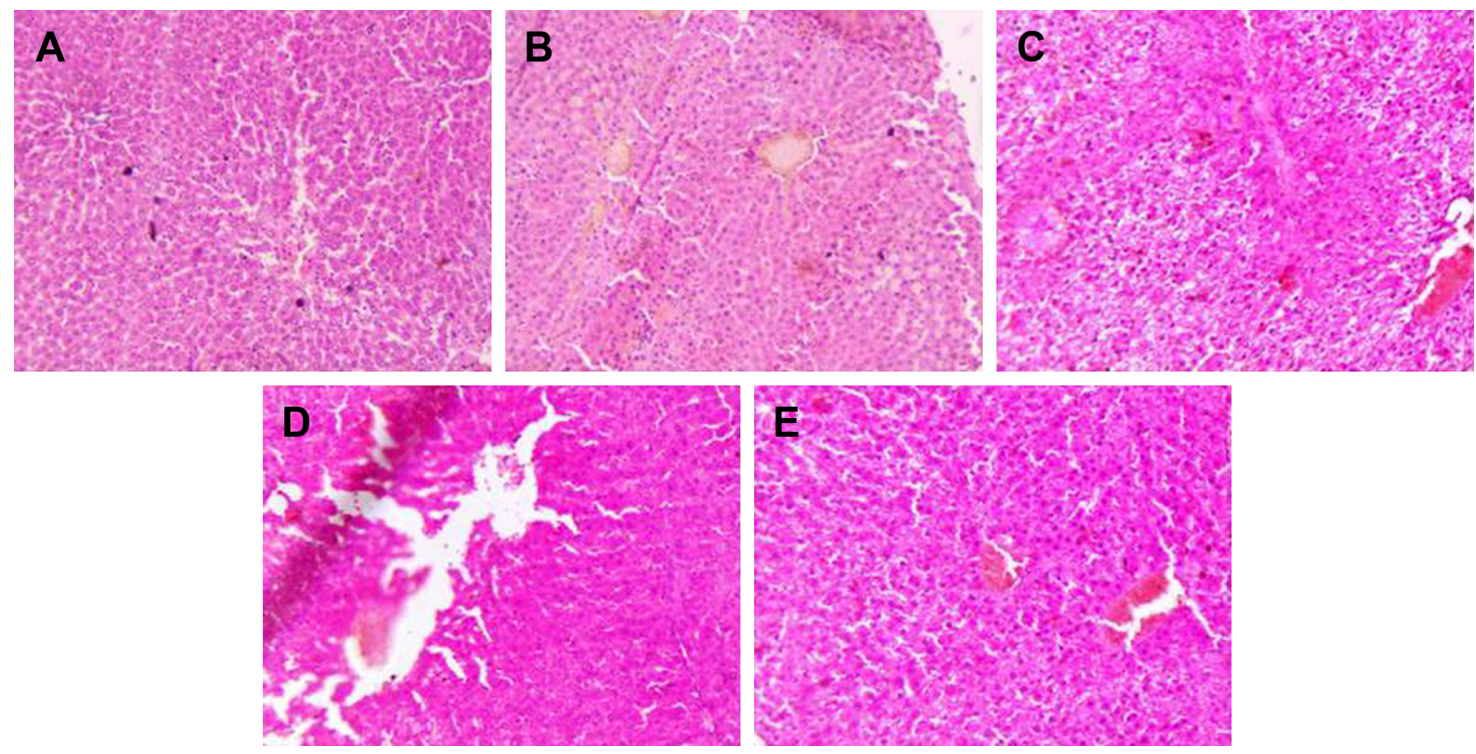

Figure 5 Effect of UFG-PLGA-NP on normal control and DEN-induced hepatocarcinogenesis rats histopathology.

Notes: (A) Normal control: rats showed the normal histopathology, (B) DEN control: showed the inflammatory blood vessels, macrodroplet of fats, cell swelling, cytoplasm with dark and irregular shaped, pseudoacini, hyperchromatic nuclei, necrosis, and disorganized hepatic parenchyma with thick cords and irregular polyhedral cells with bordering wide sinusoids, (C) DEN + UFG ( $5 \mathrm{mg} / \mathrm{kg})$ : showed the inflammatory blood cells, necrosis, irregular architecture, less altered hepatocytes, less enlarged karyomegali, proliferation in portal area of HSCs, and HSCs' focal proliferation with less macrodroplets of fats, (D) DEN + UFG (50 mg/kg): treated rats showed the less inflammatory blood vessels, cell necrosis, and less macrodroplet of fats accumulated in the rat liver, (E) DEN + UFG-PLGA-NP-treated rats showed no necrosis in the cell and blood vessels, less inflammatory blood vessels, and compact cytoplasm with normal size of karyomegali and bile duct. Original magnification I0X; DXIT I200, Nikon, Japan.

Abbreviations: DEN, diethyl nitrosamine; HSCs, hepatic stellate cells; PLGA, poly(d,I-lactide-co-glycolide); UFG, umbelliferone $\beta$-D-galactopyranoside; UFG-PLGA-NP, UFG-loaded PLGA nanoparticles.

average central vein, small uniform nuclei of cytoplasm, and normal polyhedral-shape hepatocytes (Figure 5). Cell necrosis with inflammatory blood vessels, nonsystemic hepatic parenchyma with thick cords (trabeculae), small dark cytoplasm with irregular structure, hyperchromatic nuclei, and pseudo acini was observed in DEN rats, and the observed feature confirmed the hepatic toxicity. Hepatic portion of DEN-treated rats confirmed the expansion of proliferation in the portal area of hepatic stellate cells (HSCs) and induces the focal proliferation in HSCs. Abnormal hepatocytes, which contain the irregular macro lipid droplets, binucleate, and masses of eosinophils in vacuolation surrounded by the cytoplasm, are also observed in histopathological slides. DEN rats treated with UFG confirmed the improvement in histopathological changes including less inflammatory necrosis cells, superior hepatocellular architecture, less altered hepatocytes, less or complete absence of the proliferation in the portal area of HSCs, and few microdroplets observed as compared to DEN control histopathology. The effect of UFG on the histopathological changes was dose dependent. The UFG-PLGA-NP-treated rats exhibited less inflammatory cells without microdroplets, and also contained compact cytoplasm, with an average-sized mononucleus (Figure 5).

\section{Discussion}

The current protocol was an attempt to formulate the polymeric nanoparticles with an effort to curb the expansion of hepatocarcinogenesis in Wistar rats by oral feeding of UFG-loaded polymeric nanoparticles. UFG-PLGA-NP stages higher concentration of UFG in the liver and is highly efficient in thwarting the DEN-induced HCC. The nanoparticles formulated from PLGA have sustained drug release capability and are biodegradable, nontoxic, and nonimmunogenic in nature. The two factors size and size distribution of nanoparticles take important part in the determination of stability, drug release, and cellular uptake efficiency. ${ }^{22}$ The smaller size of particle provides the greater window to release the drug into the systemic circulation via reticuloendothelial system. Cancer cells have the capability to concentrate a drug 20 times more when distributed via nanoparticulate preparation, and the underlying reason is the retention effect and increased permeability of such compounds..$^{18,19}$

HCC initiated by DEN, in rodent, is one of the most accepted experimental models for liver cancer, which allows the selection of the probable anticancer compounds on different phases of neoplastic transformation and expansion. ${ }^{20}$ Here, we have scrutinized the chemoprotective effect of nanoformulation of UFG on DEN-induced hepatocarcinogenesis 
in rats and tried to decipher the possible mechanism of action. During the study, we observed the development of the hepatic nodules in the DEN-induced rats, which were comparatively lesser in UFG-treated rats, in a dose-dependent manner. DEN-induced rats confirmed the expansion of hepatocyte nodules with the size $(3 \mathrm{~mm}){ }^{24}$ The hepatocytes that have been recognized for preneoplastic nodules for prominent hyperplasia, amendment in genes for cell proliferation, and enzymatic markers are well documented to establish the relation. Several researchers have confirmed that the development of hepatocyte nodules plays an important role as a precursor of HCC. ${ }^{19,20}$ During the course of study, the result illustrated that the nanoformulation of UFG suppressed the development of hepatic nodules and decreased the content of hepatic serum enzymes in experimental rats. ${ }^{21,22}$ The result accomplished the objective of our hypothesis by decreasing the development of hepatocyte nodules and restoring the hepatic enzyme against DEN intoxication. ${ }^{23}$ We also found that the UFG nanoformulation was relatively efficient against eliminating the hepatic toxicity induced by DEN.

AFP, considered as the gold standard marker, was used in the estimation of $\mathrm{HCC}$ along with other serum parameters. The shape, size, and composition of the AFP are similar to the albumin, but the estimation process of AFP is rapid as compared to other albumins. AFP is generally used for the estimation of hepatocarcinoma and also for evaluating tumor growth. ${ }^{24}$ The results of DEN-induced HCC group exhibited increased level of AFP, 10 times more than the normal control rats, which was significantly $(P<0.001)$ reduced by UFG nanoformulation in a dose-dependent manner. Further, our results established the preventive effect of UFG in UFG-NPtreated rats as compared to DEN control rats, by decreasing hepatic nodules, as evident from the microscopic and histopathological evaluation of each group (Figure 4). ${ }^{17}$

The ALP, ALT, and AST are the hepatic markers to judge the condition of liver. These transaminase enzymes are mostly present in heart, liver, and muscle. Any injury or damage of tissue or organ leads to oozing of these enzymes in circulating blood along with other enzyme and intercellular proteins. Determination of the serum ALT and AST levels advises the normal or altered function of liver. ${ }^{25,26}$ Functional integrity and cellular injury of the liver cell membrane can easily be scrutinized if there is enhanced content of AST and ALT in serum. DEN control group rats showed the increased level of AST and ALT and confirmed the hepatic toxicity. ${ }^{23,24}$ DEN-induced rats treated with nanoformulation of UFG significantly $(P<0.001)$ decreased the content of AST and ALT in a dose-dependent manner. The hepatoprotective nature of drug/isolated compound can be appraised by its potential to restore or protect the liver functional mechanism that was induced by DEN carcinogenesis.

Hematological parameters used to identify the interaction between the biological system and chemicals in vivo system suggest the systemic effects. RBC play an important role during the respiration; they contain $\mathrm{Hb}$, which transports the oxygen to the tissue and revert the carbon dioxide to the lungs. The reduced level of $\mathrm{RBC}$ and $\mathrm{Hb}$ indicates anemia. Another hematological parameter, WBC, defends the body against the infections. The DEN-induced HCC showed the increase in the level of WBC and suggests the enhancement of the pathological condition and immunological activity. ${ }^{32}$

Oxidative stress induced by generation of reactive nitrogen species/ROS and free radicals including peroxynitrite, superoxide, hydrogen peroxide, and hydroxyl radical is a result of injury or due to decreased content of antioxidant or inflammatory reaction via nuclear factor-kappa B $(\mathrm{NF}-\kappa \mathrm{B}) .{ }^{18,27}$ During hepatic carcinogenesis, the generation of free radicals and their reaction with lipid to produce LPO is considered as the important mechanism for corpuscle damage. The initiation of LPO starts the production of toxic radicals such as MDA, hydrogen peroxide, F2-isoprostanes, hydroxyl radicals, and 4-hydroxynonenal, which causes cell death, cell membrane damage, cellular function, oxidative stress, and carcinogenesis. ${ }^{35,36}$ The content of MDA and CD was increased during the HCC development from increased production of free radicals such as alkoxy and peroxyl radicals, and enhanced the deposition of these radicals to cause the tissue toxicity and dysfunction of endogenous antioxidant systems. The results revealed that increased level of $\mathrm{CD}$ and MDA in DEN control group was significantly declined by the nanoformulation of UFG. The possible mechanism of action of UFG may be inhibition of alkoxy and peroxyl radical production resulting in hepatoprotective effect in hepatic cancer. SOD and CAT are considered as the first-line antioxidant enzymes; SOD scavenges the superoxide to hydrogen peroxide and CAT scavenges and detoxifies the hydrogen peroxide. The restoration of these enzymes is also attributed to our formulation. ${ }^{24-28}$ Histological studies reveal that the HCC expansion occurs in the backdrop of chronic inflammation and NF- $\mathrm{KB}$ is the chief mediator of inflammation, regulating other inflammatory molecules either downstream or upstream. ${ }^{18,23,29}$ Liver is the production center for synthesis of cytokines such as TNF- $\alpha$, IL- $1 \beta$, and IL-6 by blood cells (monocytes and lymphocytes). Not only liver but also few other cells such as murine, kupffer cells, and human hepatocytes produce IL-1 $\beta$, IL-6, and TNF- $\alpha$. 
Cytokines such as IL-6, secreted from the kupffer cells via modulating the NF- $\kappa \mathrm{B}$ signaling, are MyD88 dependent, which further activate the IL- $1 \alpha$ from hepatocytes. ${ }^{37,38}$ Other cytokines such as TNF- $\alpha$ (NF- $\kappa$ B activator) have a significant role in the inflammatory reaction and also play a critical role in the cell proliferation. The possible underlying mechanism to initiate hepatocarcinogenesis by DEN may be from its effects on kupffer cells via activation of NF- $\kappa B$, which regulates the inflammatory mediators such as IL- 6 and TNF- $\alpha$ into the circulation. ${ }^{30,31}$ It also plays a dual role in the development of neoplasia, induction of necrosis, and expansion of fibroblasts and other tumor cells, and also has secondary role in demolishing the cancer vasculature. Experimental evidence suggested that TNF- $\alpha$ accelerates the production of genotoxic molecules and induces the antiapoptotic molecules that are NF- $\kappa \mathrm{B}$ dependent, which confirm its effect on tumor growth. ${ }^{30-34}$ DEN group rats treated with UFGPLGA-NP showed the decrease in the level of TNF- $\alpha$ as compared to DEN control. The possible mechanism of action of UFG-PLGA-NP may be by inhibiting the production of genotoxic molecules as well as reducing the antiapoptotic molecules that are NF- $\kappa \mathrm{B}$ dependent. In relation to cancer progress, UFG may be a beneficial chemopreventive tool but the complexity remains to determine the efficient dose and time of administration, and to maneuver the compound to the target site needs some more in-depth study.

\section{Disclosure}

The authors report no conflicts of interest in this work.

\section{References}

1. Rahman MA, Dhar DK, Yamaguchi E, et al. Coexpression of inducible nitric oxide synthase and COX-2 in hepatocellular carcinoma and surrounding liver: possible involvement of COX-2 in the angiogenesis of hepatitis C virus-positive cases. Clin Cancer Res. 2001; 7(5):1325-1332.

2. Lara-Pezzi E, Gómez-Gaviro MV, Gálvez BG, et al. The hepatitis B virus $\mathrm{X}$ protein promotes tumor cell invasion by inducing membranetype matrix metalloproteinase-1 and cyclooxygenase-2 expression. J Clin Invest. 2002;110(12):1831-1838.

3. Bhatt AN, Mathur R, Farooque A, Verma A, Dwarakanath BS. Cancer biomarkers - current perspectives. Indian J Med Res. 2010;132: 129-149.

4. Farmer DG, Seu P, Swenson K, Economou J, Busuttil RW. Current and future treatment modalities for hepatocellular carcinoma. Clin Liver Dis. 1997;1(2):361-396, ix.

5. Anwar F, Mushtaq G, Kazmi I, et al. Anticancer effect of rosiglitazone in rats treated with $\mathrm{N}$-nitrosodiethylamine via inhibition of DNA synthesis: an implication for hepatocellular carcinoma. RSC $A d v$. 2015;5:68385-68391.

6. Anwar F, Al-Abbasi FA, Bhatt PC, Ahmad A, Sethi A, Kumar V. Umbelliferone $\beta$-d-galactopyranoside inhibits chemically induced renal carcinogenesis via alteration of oxidative stress, hyperproliferation and inflammation: possible role of NF-KB. Toxicol Res. 2015;4(5): $1308-1323$.
7. Yu SM, Hu DH, Zhang JJ. Umbelliferone exhibits anticancer activity via the induction of apoptosis and cell cycle arrest in HepG2 hepatocellular carcinoma cells. Mol Med Rep. 2015;12(3):3869-3873.

8. Ghosh D, Choudhury ST, Ghosh S, et al. Nanocapsulated curcumin: oral chemopreventive formulation against diethylnitrosamine induced hepatocellular carcinoma in rat. Chem Biol Interact. 2012;195(3): 206-214.

9. Kumar V, Ahmed D, Verma A, Anwar F, Ali M, Mujeeb M. Umbelliferone $\beta$-D-galactopyranoside from Aegle marmelos (L.) corr. an ethnomedicinal plant with antidiabetic, antihyperlipidemic and antioxidative activity. BMC Complement Altern Med. 2013; 13:273.

10. Kumar V, Abbasi AF, Verma A, Mujeed M, Anwar F. Umbelliferone $\beta$-d-galactopyranoside exerts an anti-inflammatory effect by attenuating COX-1 and COX-2. Toxicol Res. 2015;4(4):1072-1084.

11. Perlatti B, de Souza Bergo PL, Fernandes da Silv MFG, Fernandes JB, Forim M. Polymeric nanoparticle-based insecticides: a controlled release purpose for agrochemicals. Insectic Dev Safer More Eff Technol. 2013:523-550.

12. Verma A, Bhatt PC, kaithwas G, et al. Chemomodulatory effect Melastoma Malabathricum Linn against chemically induced renal carcinogenesis rats via attenuation of inflammation, oxidative stress, and early markers of tumor expansion. Inflammopharmacology. 2016; 24(5):233-251.

13. Kumar V, Bhatt PC, Kaithwas G, et al. $\alpha$-mangostin mediated pharmacological modulation of hepatic carbohydrate metabolism in diabetes induced Wistar. Beni Suef University Journal of Basis and Applied Sciences. 2016;5(3):255-276.

14. Kumar V, Al-Abbasi FA, Ahmed D, Verma A, Mujeeb M, Anwar F. Paederia foetida Linn. inhibits adjuvant induced arthritis by suppression of PGE(2) and COX-2 expression via nuclear factor-kB. Food Funct. 2015;6(5):1652-1666.

15. Verma A, Singh D, Bhatt PC, Anwar F, Al-Abbasi FA, Kumar V. Triterpenoids principle of Wedelia calendulacea attenuated diethynitrosamine-induced hepatocellular carcinoma via down-regulating oxidative stress, inflammation and pathology via NF-kB pathway. Inflammopharmacology. Epub 2017 Jun 12.

16. Kumar V, Anwar F, Verma A, Mujeeb M. Therapeutic effect of umbelliferon- $\alpha$-D-glucopyranosyl-(2(I) $\rightarrow 1$ (II))- $\alpha$-D-glucopyranoside on adjuvant-induced arthritic rats. J Food Sci Technol. 2015; 52(6):3402-3411.

17. Khan R, Kazmi I, Afzal M, et al. Fixed dose combination therapy loperamide and niacin ameliorates diethylnitrosamine-induced liver carcinogenesis in albino Wistar rats. RSC Adv. 2015;5(83): 67996-68002.

18. Afzal M, Kazmi I, Khan R, et al. Thiamine potentiates chemoprotective effects of ibuprofen in DEN induced hepatic cancer via alteration of oxidative stress and inflammatory mechanism. Arch Biochem Biophys. 2017;623-624:58-63.

19. Kumar V, Anwar F, Ahmed D, et al. Paederia foetida Linn. leaf extract: an antihyperlipidemic, antihyperglycaemic and antioxidant activity. BMC Complement Altern Med. 2014;14:76.

20. Verma A, Ahmad B, Anwar F, et al. Novel glycoside from Wedelia calendulacea inhibit Diethyl nitrosamine induced renal cancer via downregulating the COX-2 and PEG2 through nuclear factor- $\mathrm{\kappa B}$ pathway. Inflammopharmacology. 2017;25(1):159-175.

21. Mandal AK, Ghosh D, Sarkar S, Ghosh A, Swarnakar S, Das N. Nanocapsulated quercetin downregulates rat hepatic MMP-13 and controls diethylnitrosamine-induced carcinoma. Nanomedicine (Lond). 2014; 9(15):2323-2337.

22. Pham CT. Nanotherapeutic approaches for the treatment of rheumatoid arthritis. Wiley Interdiscip Rev Nanomed Nanobiotechnol. 2011; 3(6):607-619.

23. Pradeep K, Mohan CV, Gobianand K, Karthikeyan S. Silymarin modulates the oxidant-antioxidant imbalance during diethylnitrosamine induced oxidative stress in rats. Eur J Pharmacol. 2007;560(2-3): $110-116$. 
24. Bishayee A, Bhatia D, Thoppil RJ, Darvesh AS, Nevo E, Lansky EP. Pomegranate-mediated chemoprevention of experimental hepatocarcinogenesis involves Nrf2-regulated antioxidant mechanisms. Carcinogenesis. 2011;32(6):888-896.

25. Chuang SE, Cheng AL, Lin JK, Kuo ML. Inhibition by curcumin of diethylnitrosamine-induced hepatic hyperplasia, inflammation, cellular gene products and cell-cycle-related proteins in rats. Food Chem Toxicol. 2000;38(11):991-995.

26. Balamurugan K, Karthikeyan J. Evaluation of luteolin in the prevention of $N$-nitrosodiethylamine-induced hepatocellular carcinoma using animal model system. Ind J Clin Biochem. 2012;27(2):157-163.

27. Liu Z, Kullman SW, Bencic DC, Torten M, Hinton DE. Ras oncogene mutations in diethylnitrosamine-induced hepatic tumors in medaka (Oryzias latipes), a teleost fish. Mutat Res. 2003;539(1-2):43-53.

28. Luther DJ, Ohanyan V, Shamhart PE, et al. Chemopreventive doses of resveratrol do not produce cardiotoxicity in a rodent model of hepatocellular carcinoma. Invest New Drugs. 2011;29(2):380-391.

29. Matsumoto K, Huang J, Viswakarma N, et al. Transcription coactivator PBP/MED1-deficient hepatocytes are not susceptible to diethylnitrosamine-induced hepatocarcinogenesis in the mouse. Carcinogenesis. 2010;31(2):318-325.

30. Jagan S, Ramakrishnan G, Anandakumar P, Kamaraj S, Devaki T. Antiproliferative potential of gallic acid against diethylnitrosamineinduced rat hepatocellular carcinoma. Mol Cell Biochem. 2008; 319(1-2):51-59.

31. Kang JS, Wanibuchi H, Morimura K, Gonzalez FJ, Fukushima S. Role of CYP2E1 in diethylnitrosamine-induced hepatocarcinogenesis in vivo. Cancer Res. 2007;67(23):11141-11146.
32. Chidambara Murthy KN, Jayaprakasha GK, Singh RP. Studies on antioxidant activity of pomegranate (Punica granatum) peel extract using in vivo models. J Agric Food Chem. 2002;50(17):4791-4795.

33. Pradeep K, Raj Mohan CV, Gobianand K, Karthikeyan S. Protective effect of Cassia fistula Linn. on diethylnitrosamine induced hepatocellular damage and oxidative stress in ethanol pretreated rats. Biol Res. 2010;43(1):113-125.

34. Gupta C, Tripathi DN, Vikram A, Ramarao P, Jena GB. Quercetin inhibits diethylnitrosamine-induced hepatic preneoplastic lesions in rats. Nutr Cancer. 2011;63(2):234-241.

35. Bingül I, Başaran-Küçükgergin C, Tekkeşin MS, Olgaç V, DoğruAbbasoğlu S, Uysal M. Effect of blueberry pretreatment on diethyInitrosamine-induced oxidative stress and liver injury in rats. Environ Toxicol Pharmacol. 2013;36(2):529-538

36. Shaban NZ, El-Kersh MA, El-Rashidy FH, Habashy NH. Protective role of Punica granatum (pomegranate) peel and seed oil extracts on diethylnitrosamine and phenobarbital-induced hepatic injury in male rats. Food Chem. 2013;141(3):1587-1596.

37. Choi EJ, Lee S, Chae JR, Lee HS, Jun CD, Kim SH. Eupatilin inhibits lipopolysaccharide-induced expression of inflammatory mediators in macrophages. Life Sci. 2011;88(25-26):1121-1126.

38. Bishayee A, Waghray A, Barnes KF, et al. Suppression of the inflammatory cascade is implicated in resveratrol chemoprevention of experimental hepatocarcinogenesis. Pharm Res. 2010;27(6): 1080-1091.
International Journal of Nanomedicine

\section{Publish your work in this journal}

The International Journal of Nanomedicine is an international, peerreviewed journal focusing on the application of nanotechnology in diagnostics, therapeutics, and drug delivery systems throughout the biomedical field. This journal is indexed on PubMed Central, MedLine, CAS, SciSearch ${ }^{\circledR}$, Current Contents ${ }^{\circledR} /$ Clinical Medicine,

\section{Dovepress}

Journal Citation Reports/Science Edition, EMBase, Scopus and the Elsevier Bibliographic databases. The manuscript management system is completely online and includes a very quick and fair peer-review system, which is all easy to use. Visit http://www.dovepress.com/ testimonials.php to read real quotes from published authors. 